\title{
QUASE-VERDADE: ASPECTOS PRAGMÁTICOS E FORMALIZAÇÃO
}

Douglas Alexandre Rodrigues

Possui graduação em Matemática pela Universidade Federal de São Carlos (2003). Especialização em Matemática Universitária pela Universidade Estadual Paulista Júlio de Mesquita Filho (2008). Atualmente é professor do Instituto Federal do Paraná e mestre em Educação Matemática pela Universidade Estadual Paulista Júlio de Mesquita Filho (2014). Atualmente, é mestrando em Filosofia pela FFC - UNESP, Campus de Marília. Email: douglas.rodrigues@ifpr.edu.br

\section{RESUMO}

O objetivo deste estudo é discutir os aspectos pragmáticos da concepção de verdade e analisar a lógica subjacente à estrutura matemática na teoria da quaseverdade, propondo um outro tipo de sistema dedutivo para essa lógica. Entretanto, faz-se necessário, inicialmente, uma reflexão acerca do papel da verdade na filosofia da ciência, pois, de acordo com a concepção correspondencial de verdade, uma sentença, podendo exprimir uma crença, é verdadeira ao retratar algo real, caso contrário é falsa. Neste caso, a crença ou sentença indica se estados de coisas existem, sendo assim, verdadeiros, caso contrário falso. Ademais, Alfred Tarski em seu artigo $O$ conceito de verdade em linguagens formalizadas, apresenta a hipótese de que 'verdadeiro' ou 'falso' não representa uma predicação de objetos, mas precisamente atributos de sentenças. Sendo assim, Tarski estabelece uma construção na qual predica esses atributos sem considerar uma auto-referência contraditória. Neste caso, em que sentido o conceito de verdade pragmática generaliza a verdade correspondencial de Tarski em situações de parcialidade? Segundo Peirce, a verdade representa o fim da indagação científica quando esta nos conduzir a um sistema de crenças estável, e, com o passar do tempo e o progresso científico, teremos atingido a verdade. A aproximação entre o pragmatismo de Peirce e o conceito de verdade de Tarski parece patente ao analisarmos a formalização da estrutura pragmática da quaseverdade. Pesquisas recentes sobre a quase-verdade mostram que a lógica adequada para teorias que envolvem uma situação que se aproxima da verdade é uma lógica paraconsistente, apresentada como um sistema axiomático. Além disso, outros trabalhos investigam a Lógica da Verdade Pragmática (LPT) com a proposta de interpretar a LPT por meio de outros sistemas dedutivos. Assim, do ponto de vista lógico, torna-se necessário investigar possíveis sistemas dedutivos para a Lógica da Verdade Pragmática aplicadas à noção de quase-verdade e propor algum outro tipo de sistema, pois tais questões parecem fundamentais para os novos paradigmas na Filosofia e na Ciência. 
PALAVRAS-CHAVE: Teorias da Verdade. Pragmatismo. Lógica.

\title{
NEAR-TRUTH: PRAGMATIC ASPECTS AND FORMALIZATION
}

\begin{abstract}
The objective of this project is to discuss the pragmatic aspects of the conception of truth and to analyze the logic underlying the mathematical structure in the theory of quasi-truth, proposing another type of deductive system for this logic. However, it is necessary, initially, a reflection on the role of truth in the philosophy of science, because, according to the correspondent conception of truth, a sentence, expressing a belief, is true when portraying something real, otherwise it is false In this case, the belief or sentence indicates whether states of things exist, thus being true, otherwise false. In addition, according to Alfred Tarski in his article The concept of truth in formalized languages presents the hypothesis that 'true' or 'false' does not represent a predication of objects, but precisely attributes of sentences. Thus, Tarski establishes a construct in which he preaches these attributes without considering a self-contradictory self-reference. In this case, in what sense does the concept of pragmatic truth generalize the truth correspondence of Tarski in situations of partiality? According to Pierce, truth represents the end of scientific inquiry when it leads us to a stable belief system, and, over time and scientific progress, we have reached the truth. The approximation between Pierce's pragmatism and Tarski's concept of truth seems clear when we analyze the formalization of the pragmatic structure of quasi-truth. Recent research on quasi-truth shows that the appropriate logic for theories involving a situation approaching truth is a paraconsistent logic presented as an axiomatic system. In addition, other works investigate the Pragmatic Truth Logic (LPT) with the proposal to interpret the LPT through other deductive systems. Thus, from a logical point of view, it becomes necessary to investigate possible deductive systems for the Pragmatic Truth Logic applied to the notion of quasitruth and to propose some other kind of system, since such questions seem fundamental to the new paradigms in Philosophy and Science.
\end{abstract}

KEYWORDS: Theories of Truth. Pragmatism. Logic.

\section{INTRODUÇÃO}

O objetivo deste trabalho é esboçar os aspectos pragmáticos da teoria da quase-verdade e apresentar algumas noções sobre sua estrutura parcial. Inicialmente, a verdade pragmática de Newton da Costa parece ter tido sua motivação na teoria da verdade correspondencial de Alfred Tarski e no pragmatismo de Peirce, pois aquele afirma que a verdade correspondencial relaciona verdade com realidade e este, que a verdade representa o fim da investigação, quando a opinião na qual aqueles que usam o método científico concordam entre si. Tarski argumenta, na teoria dos modelos, que as porções da realidade são substituídas por estruturas conjuntistas e 
linguagens naturais por linguagens formalizadas. Além disso, a verdade pragmática, de acordo com Peirce, James e Dewey, combinam elementos de correspondência e coerência, pois segundo os autores, o significado de um conceito refere-se às consequências experimentais ou práticas de sua aplicação. Através do aspecto pragmático da quase-verdade, busca-se capturar representações parciais da realidade. Sendo assim, a concordância entre partes da realidade e suas respectivas estruturas parciais, parece atribuir uma generalização da verdade correspondencial.

A ideia de estruturas parciais e suas correspondências com a quase-verdade possui relações no domínio que não são totais, ou seja, para cada relação teríamos que atribuir uma terna de relações. Neste caso, a pertinência de uma n-upla do domínio na relação não está sempre definida e suas fórmulas atômicas são interpretadas como uma relação $R$ definida como uma terna ordenada $\left(R_{+}, R_{-}, R_{u}\right)$ em que $R_{+}$é o conjunto das n-uplas as quais pertencem a $R, R_{-}$ é o conjunto das n-uplas as quais não pertencem a $R$ e $R_{u}$ é o conjunto das n-uplas na qual a pertinência a $R$ é indeterminada.

Atualmente, algumas investigações sobre o tema mostram que a abordagem de predicados como ternas podem ser estendidos recursivamente para toda fórmula da linguagem de primeira ordem e a lógica subjacente à teoria da quase-verdade é uma lógica paraconsistente.

\section{MALGUMAS CARACTERÍSTICAS DA CONCEPÇÃO SEMÂNTICA DA VERDADE DE TARSKI}

A concepção semântica da verdade de Alfred Tarski propõe capturar a definição aristotélica clássica da verdade: “dizer daquilo que é que não é, ou daquilo que não é que é, é falso, enquanto dizer daquilo que é que é, ou daquilo que não é que não é, é verdadeiro. De acordo com Tarski, uma definição satisfatória da verdade deve ser formalmente correta e materialmente adequada para uma 'sentença verdadeira'.

$\mathrm{Na}$ defininiçãa de verdade materialmente adequada, Tarski propõe condições mínimas de aceitabilidade nas quais seus métodos podem ser aplicados em diversos tipos de linguagens formalizadas. Sendo assim, qualquer definição materialmente adequada de verdade deve ter como consequência as instâncias do esquema T: S é verdadeira se e somente se p.

O termo em p pode ser substituído por qualquer sentença da linguagem para a qual a sentença está sendo definida em ' $\mathrm{S}$ ', e, substituído pelo nome da sentença que substituí 'p'. Uma instância de T é dada por: 'a neve é branca' é verdadeira se e seomente se a neve é branca. 
Neste caso, a adequação material deve implicar todas as instâncias do esquema T, garantindo a definição de verdade e este, ao ser aceito, fixa a extensão do temo 'verdadeiro'.

No paradoxo do mentiroso, ao afirmar 'esta sentença é falsa', Tarski investiga alguns detalhes e argumenta que a antinomia surge com as seguintes pressuposições:

1) Que a linguagem utilizada contém, além de suas expressões; os meios para se referir as expressões e predicados semânticos, tais como 'verdadeiro' e 'falso'. Tal linguagem Tarski denomina semanticamente fechada.

2) Que as leis da lógica valem (HAACK, 1998, p. 147).

Deste modo, Tarski afirma que uma definição formalmente correta de verdade deveria ser expressa em uma linguagem semanticamente fechada, ou seja, isto significa que a definição de verdade na linguagem objeto $\mathrm{O}$ (linguagem para a qual a verdade está sendo definida) terá de ser dada em uma metalinguagem M (linguagem na qual a verdade é definida em $\mathrm{O}$ ). Através dessas duas condições gerais de definição de verdade, Tarski introduza a noção de satisfatibilidade sobre o termo 'verdadeiro'.

Informalmente, o conceito de satisfação é especificado por uma estrutura sintática, $\mathrm{O}$, para a qual a linguagem deve ser definida e, também, por uma estrutura $M$, na qual a verdade em $\mathrm{O}$ deve ser definida. Além disso, $\mathrm{M}$ deve conter; ou expressões de $\mathrm{O}$, ou traduções das expressões de $\mathrm{O}$; um vocabulário sintático, incluindo os nomes dos símbolos primitivos de $\mathrm{O}$, um sinal de concatenação e variáveis para expressões de O. A satisfatibilidade é definida na linguagem $\mathrm{O}$ e a definição de verdadeiro em O em termos de satisfação em O (HAACK, 1998, p. 150).

A noção de satisfação parece apropriada, pois os termos podem ser definidos como 'verdadeiros' nos quais sentenças compostas fechadas são formadas a partir de sentenças abertas. Tarski considera que o conceito de verdade é o conceito que se aplica a sentenças, mas como proceder com funções sentenciais? $\quad$ Neste caso, as sentenças podem ser compostas por componentes atômicas que são funções sentenciais e, para funções sentenciais, o conceito de verdade não se aplica, mas pode ser satisfeita ou não por objetos. Por exemplo, ' $(\forall x)(F x \rightarrow G x)^{\prime}$ é formada a partir de 'Fx' e 'Gx' pelas operações de implicação e quantificador universal, e as sentenças abertas ' $F x$ ' e ' $G x$ ' não são nem verdadeiras e nem falsas.

A definição de satisfação é recursiva, ou seja, definida para os casos mais simples (sentenças atômicas) e, posteriormente, para a determinação do valor de verdade das 
componentes atômicas de uma fórmula. Contudo, em alguns casos, as componentes atômicas de uma sentença podem ser funções sentenciais, logo, o conceito de verdade não se aplica, mas pode ser satisfeita por objetos.

A satisfação é uma relação entre sentenças abertas e n-uplas de objetos, porém, as sentenças podem ter qualquer número de variáveis livres. Tarski estabelece a relação entre sentenças abertas e sequências infinitas, convencionando que ' $\mathrm{F}\left(x_{1}, \ldots, x_{n}\right)$ ' deve ser satisfeita pela sequência $\left\langle O_{1}, \ldots, O_{n}, O_{n+1}, \ldots>\right.$ nos casos que são satisfeitos pelos n primeiros termos da sequência (HAACK, 1998, p. 151).

$\mathrm{Na}$ explicação formal sobre o conceito de sentença verdadeira, Tarski apresenta a construção da definição usando a linguagem de cálculo de classes, porém, posteriormente, defini-se formalmente o conceito de verdade no cálculo de predicados de primeira ordem. Contudo, não apresentaremos as características técnicas da formalização do conceito de verdade em Tarski, pois focaremos nas suas relações com as estruturas parciais da quase-verdade.

\section{A QUASE-VERDADE DE NEWTON DA COSTA}

Ao analisarmos a concepção semântica de Tarski, esta poderia capturar aspectos da 'verdade' na linguagem natural? O autor enfatiza que, ao tentar definir semanticamente a verdade para a linguagem natural, acaba encontrando diversos obstáculos e segue suas investigações para as linguagens formalizadas apontando suas vantagens. Desta forma, apesar do avanço tanto na lógica, quanto na filosofia, não é rica o suficiente para capturar todas as características da verdade (FRENCH, 2003, p. 24).

Apoiado nas ideias dos filósofos pragmatistas C. S. Peirce, W. James e J. Dewey, surge um novo tipo de teoria da verdade priorizada pela experiência e a ação sobre o ser e o pensamento. De acordo com da Costa (1997) a teoria da verdade, apoiada na ideias pragmáticas, foi batizada de quase-verdade e seu conceito de verdade é estabelecido quando uma sentença $S$ é pragmaticamente verdadeira (ou quase-verdadeira) em um domínio do saber $D$. Em algumas situações, $S$ é quase-verdadeira pois $S$ é verdadeira no sentido correspondencial, ou seja, quando $S$ é uma sentença observacional. Essas ideias motivaram definir uma verdade que se aproximasse da realidade dos fatos e, juntamente com a noção de estrutura parcial, poderia ser representada formalmente.

As noções de quase-verdade e estruturas parciais foram introduzdas por Mikenberg, Da Costa e Chuaqui (1986) nas quais investiga-se certo domínio de conhecimento $\Delta$ e suas relações 
entre os objetos reais de um conjunto $D$, que pode ser representado por uma informação que possuímos sobre $\Delta$.

Para sistematizar o conhecimento de $\Delta$ é conveniente introduzir a estrutura $<$ $A_{1}, R_{i},>_{i \in I}$ e objetos denotados por $A_{2}$, tal que $A_{1} \cap A_{2}=\emptyset$. Sendo assim, a modelagem de $\Delta$ envolve novas relações parciais $R_{j} \operatorname{com} j \in J$, algumas das quais estendem as relações $R_{i} \operatorname{com} i \in I$.

Uma estrutura pragmática simples é um estrutura conjuntista da seguinte forma: $A=$ $<A_{1}, A_{2}, R_{i}, R_{j}, P>_{i \in I, j \in J}$, onde os conjuntos não-vazios $A_{1}$ e $A_{2}$, as relações parciais $R_{i}$, $i \in I, R_{j}, j \in J$ e as coleções de sentenças P satisfazem as condições precedentes.

Definição 1. Uma estrutura pragmática simples (EPS) é qualquer estrutura $D=\left\langle A, R_{k}, P\right\rangle_{k \in K}$ , onde A é um conjunto não vazio, $R_{k}$ é uma relação parcial definida sobre A para todo $k \in K$, e P é a uma coleção de sentenças da linguagem L.

Os símbolos de L são símbolos lógicos, símbolos auxiliares, uma coleção de constantes individuais e uma coleção de símbolos de predicados. Interpretar L, na EPS $D=<$ $A, R_{k}, P>_{k \in K}$ é associar a cada constante individual de $\mathrm{L}$ um elemento do universo de $\mathrm{D}$, e a cada símbolo de $\mathrm{L}$, de aridade $\mathrm{n}$, a relação $R_{k}, k \in K$, de mesma aridade.

Definição 2. Sejam L e $D=<A, R_{k}, P>_{k \in K}$ respectivamente uma linguagem e uma EPS, tais que L é interpretada em D. Seja B uma estrutura total, ou seja, estrutura usual cujas relações de aridade $\mathrm{n}$ acham-se definidas para todas as n-uplas de elementos de seu universo, e admitimos também que L esteja interpretada em B. Então diz-se A-normal se as seguintes condições são satisfeitas:

1) $O$ universo de $B$ é $A$;

2) As relações totais de B estendem as relações correspondentes de A;

3) Se c for uma constante individual de L, em D e B, c é interpretada pelo mesmo elemento;

4) Se $\alpha$ for uma sentença de P, $\alpha$ é verdadeira na estrutura B (DA COSTA, 1997, p.130-131).

Definição 3. Sejam L e D respectivamente uma linguagem e uma estrutura pragmática simples, na qual L está interpretada. Dizemos que a sentença $\alpha$ de L é pragmaticamente verdadeira na EPS D, de acordo com B, se B for uma estrutura A-normal e $\alpha$ for verdadeira em B, segundo a definição de Tarski.

Dizemos que $\alpha$ é pragmaticamente verdadeira na EPS D se existir uma estrutura Anormal, B, na qual $\alpha$ é verdadeira.Se $\alpha$ não é pragmaticamente verdadeira na EPS D, de acordo 
com B, diz-se que é pragmaticamente falsa na EPS D, de acordo com B (DA COSTA, 1997, p.130-131).

A teoria da quase-verdade possibilita acomodar a parcialidade informacional encontrada na ciência empírica. De acordo com Bueno (1999), se uma sentença é pragmaticamente verdadeira, ela descreve o domínio particular sob consideração como se sua descriação fosse verdadeira. Sendo esta consistente com o conhecimento básico presente no domínio em exame, tal sentença permite a apreensão de algumas das principais informações a respeito deste último, sem comprometer-nos com a aceitação da verdade dos demais itens de informação (formulados pela estrutura total A-normal). Essa definição representa uma generalização da definição tarskiana de verdade, com relação às estruturas parciais, pois ambas as definições são exatamente as mesmas quando a primeira é restrita a estruturas totais.

\section{CONSIDERAÇÕES FINAIS}

Apresentamos aspectos gerais da formalização da quase-verdade e suas relações com a concepção semântica de Tarski. Recentemente, algumas pesquisas mostram uma nova abordagem para a noção de quase-verdade, apresentando a noção de predicados como triplas definida recursivamente para toda linguagem objeto de primeira ordem. Desta forma, tal proposta generaliza a noção de verdade de Tarski e a noção de quase-verdade de Newton da Costa.

Coniglio e Silvestrini (2014) apresentam uma nova abordagem na axiomatização da noção de quase-verdade e obtêm uma lógica paraconsistente trivalorada como base proposicional utlizando uma lógica de primeira ordem. Por meio deste estudos e artigos relacionados com a quase-verdade, pretendemos investigar os aspectos formais de uma lógica adequada para teoria da quase-verdade. Além disso, torna-se relevante pesquisar possíveis sistemas dedutivos para a Lógica da Verdade Pragmática (LPT) aplicados à noção de quaseverdade. 


\section{REFERÊNCIAS BIBLIOGRÁFICAS}

BUENO, O. Empirismo Construtivo, Centro de Lógica, Epistemologia e História da Ciência - Campinas Unicamp, 1999.

CONIGLIO, M. E. \& SILVESTRINI, L. H. C. An alternative approach for quasi-truth. Logic Journal of the IGPL, v. 22, p. 387-410, 2014.

da COSTA N. C. A. O Conhecimento Científico, 2 ed. São Paulo: Discurso Editorial. 1999. da COSTA, N. C. A. O., FRENCH S. Science and Partial Truth, Oxford Universty Press, 2003.

HAACK, S. Filosofia das Lógicas, Ed, Unesp, 1998.

MIKENBERG, I., da COSTA, N. C. A., and R. CHUAQUI. Pragmatic Truth and Approximation to Truth. The Journal of Symbolic Logic, 51-1:201$221,1986$.

RODRIGUES, D.A. Quase-Verdade: Aspectos Pragmáticos e Formalização. Complexitas - Rev. Fil. Tem. Belém, v. 3, n. 1, p. 161-168, jan./jun. 2018. Disponível em:< http://www.periodicos.ufpa.br/index.php/complexitas/article/view/6630>. Acesso em: 20 de fevereiro de 2019. 